\title{
A tabletop line-of-sight stabilization demonstrator for STEM outreach activities
}

Brennan Taylor, Evan Threlkeld, Tyler Brewer, J. D. Vera Cruz, Matthew Cooper

Brennan Taylor, Evan Threlkeld, Tyler Brewer, J. D. Vera Cruz, Matthew Cooper, "A tabletop line-of-sight stabilization demonstrator for STEM outreach activities," Proc. SPIE 11143, Fifteenth Conference on Education and Training in Optics and Photonics: ETOP 2019, 111431D (2 July 2019); doi: $10.1117 / 12.2521811$

SPIE Event: Fifteenth Conference on Education and Training in Optics and Photonics: ETOP 2019, 2019, Quebec City, Quebec, Canada 


\title{
A Tabletop Line-Of-Sight Stabilization Demonstrator for STEM Outreach Activities
}

\author{
Brennan Taylor, Evan Threlkeld, Tyler Brewer, JD Vera Cruz, and Matthew Cooper \\ Air Force Research Laboratory, 3550 Aberdeen Ave SE, Kirtland AFB, NM 87112, USA
}

\begin{abstract}
The Air Force Research Laboratory's Laser Division faces the unique challenge of explaining intricate technical problems in its research portfolio to a varied audience, ranging from upper military leadership in control of program funding to young elementary students involved in STEM outreach activities. A team of Lieutenants from Kirtland Air Force Base developed an innovative solution to this challenge with a series of tabletop demos used to visually illustrate some of the key technical challenges and fundamental science the lab is working on. The negative effects of vibration on a laser system is a visually apparent problem and thus jitter was the focus of our first demo. The demo uses a Line-of-Sight Stabilization system to stabilize a vibrating laser beam. This paper will discuss the system design, implementation of the control loop, presentation techniques, and opportunities for expansion. This demo boasts a unique ability to speak to all ages, making it easier to explain key concepts to military leadership at the Pentagon as well as expose students across the USA to STEM challenges. This system is also one of a two-part set. The other, focusing on Adaptive Optics, is discussed in a sister paper.
\end{abstract}

Keywords:

Education, STEM, Lasers, Optics, Vibration, Fast-Steering-Mirrors, Position-Sensing Detector, Stabilization, Feedback, Control Theory

\section{INTRODUCTION}

The Line of Sight (LOS) Stabilization Demonstrator is an interactive, tabletop visualization tool that shows the effects of vibration on a laser beam as well as a basic stabilization technique. The beam from a green laser diode is bounced off a vibration-susceptible mirror and a Fast Steering Mirror (FSM), before passing through a beamsplitter which sends approximately $10 \%$ of the light into a Position Sensing Detector (PSD). The PSD is linked in a feedback loop to the FSM. Thus, when engaged, the two work in tandem to stabilize the beam.

The system is mounted on a small optical breadboard, allowing for easy portability and has been demonstrated with audiences large and small. One of the best ways of teaching an audience about lasers is to use a visual example. This system is simple enough that nearly any observer can understand how it works with only a brief explanation. However, it encapsulates several advanced technical challenges which allows for deeper explanation given an astute audience.

Section 2 of this paper describes the generation of the LOS Demo idea and the story we tell with this demo. It also presents several descriptions and pictures from events this demo has supported. Section 3 describes the system design and several lessons learned from construction and subsequent modifications. A full parts list of everything used in the current design of the system is included as an Appendix. Finally, Section 4 concludes by describing two different modifications of the system: how a well-funded user could expand it capabilities, and a few options for how the classroom teacher on a budget can show a similar visualization and apply these principles to primary and secondary educational environments. 


\section{Overview of the LOS Demo Development}

The LOS Demonstrator was created as part of the response to a challenge presented to our original design team. We were tasked with creating an interesting, approachable tour concept for our facility. Our building is one of the most common stops for any tour group who visited the Air Force Research Laboratory at Kirtland AFB but our specific program lacked a concise way to explain our work to those interested in what we did. Our team was given one month to design and present a new tour concept that could showcase the great work we were doing to a wide variety of audiences.

From the initial stages of this project, we quickly recognized our work represents a rather technically complex and unique problem at the intersection of optics, laser physics, and aerodynamics. Very few of our potential audiences would have a scientific background in any one of these fields, much less all of them. Thus, we would need to distill the important content down to a level that, while still being technically accurate, would also be understandable by anyone regardless of their scientific background. We tossed around a few ideas for a video or storyboard but ultimately came to the conclusion that there is nothing quite like observing a scientific phenomenon occur right before your eyes. Thus, the demo tables were born. To narrow our focus for what we wanted to present, we focused on two of the most fundamental technical challenges we face: jitter and aero-optical disturbances. This paper focuses on the first of those challenges.

Jitter is the term given to beam motion as a result of platform and environmental vibration and represents a major technological challenge for any laser weapons system. Laser weapons work by depositing energy into a small point on a target and thus causing a physical effect. If the beam is subjected to high amounts of jitter, the beam spot moves rapidly back and forth across the target and the energy is spread out rather than concentrated on a single aimpoint, thus dramatically increasing the time required to achieve the desired effect.

As you are probably realizing, it would be much easier to simply see a beam vibrating than to try to understand the previous description! Anyone can watch a laser beam and tell if it is moving in a way that is unintentional; however, start throwing out terms such as jitter, aimpoint, dwell time, and stabilization while people are looking at a complicated optical system set up on laboratory bench and their eyes will glaze over before you finish your first sentence. Thus, our story with this demo starts at the most fundamental level: beam vibrates, beam does not vibrate.

Our team has found for most audiences, the best way to hook viewers' interest is to start by showing how vibration affects the beam spot size, then switching on the control loop. Observing the beam suddenly snap into a stabilized position while the vibration continues unchanged is all most viewers need to discover their desire to know what's going on. From that point it is rather simple to walk them through the demo, highlighting the PSD which senses the displacement, and the FSM which applies the correction.

As mentioned previously, this demo allows tailored explanations for the astute observer. Due to the military nature of our work, we commonly receive questions about target tracking, i.e. “do you use this to track a flying target?” This leads to a discussion about the differences between a beam director (pointing a beam in a desired direction beyond the platform) and this feedback control loop (small scale alignment correction within the platform). We also use this demo to illustrate how several different lasers make up a laser weapon system, each with a different purpose and design. Tracking lasers operate as spotlights, highlighting the target for tracker cameras. Stabilization lasers operate exactly as demonstrated with this kit: using low power beams to measure the vibration inside the system and apply a correction. Finally, a High Energy Laser (HEL) is used to cause a damaging effect on the target after receiving all of the corrections determined by the other systems. This tends to lead into a discussion about packaging: how we get all of that in a small space, with low weight, and small enough power requirement to fly it on Air Force aircraft. Thus, while students can walk away fascinated by a simple example of an analog optical control loop, we also have the tools to discuss some of the more intricate details of our research with other scientists, military leadership, and other interested parties. 


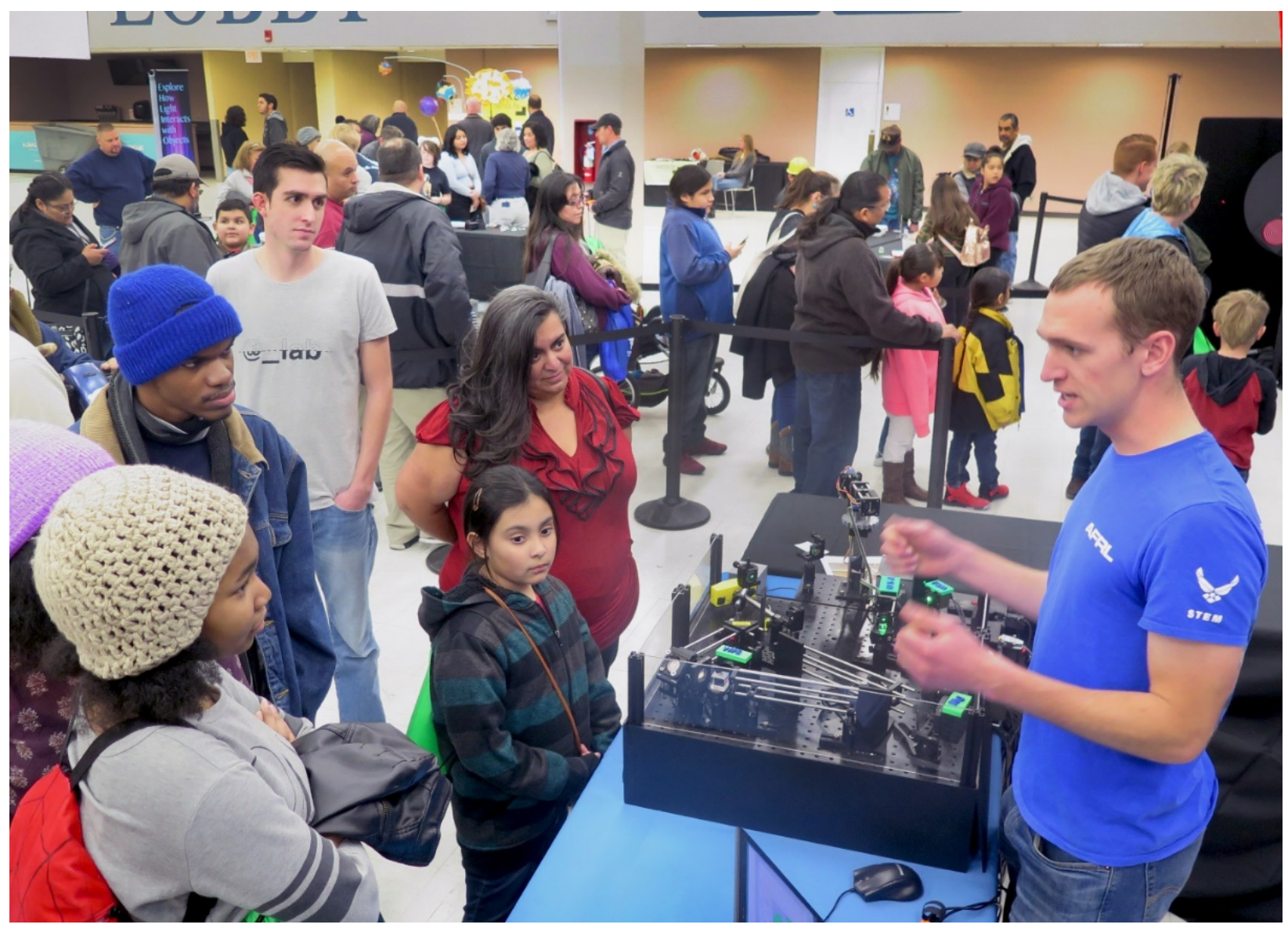

Figure 1. Lt Brewer explains how the demo works at Super STEM Saturday in Albuquerque, NM 


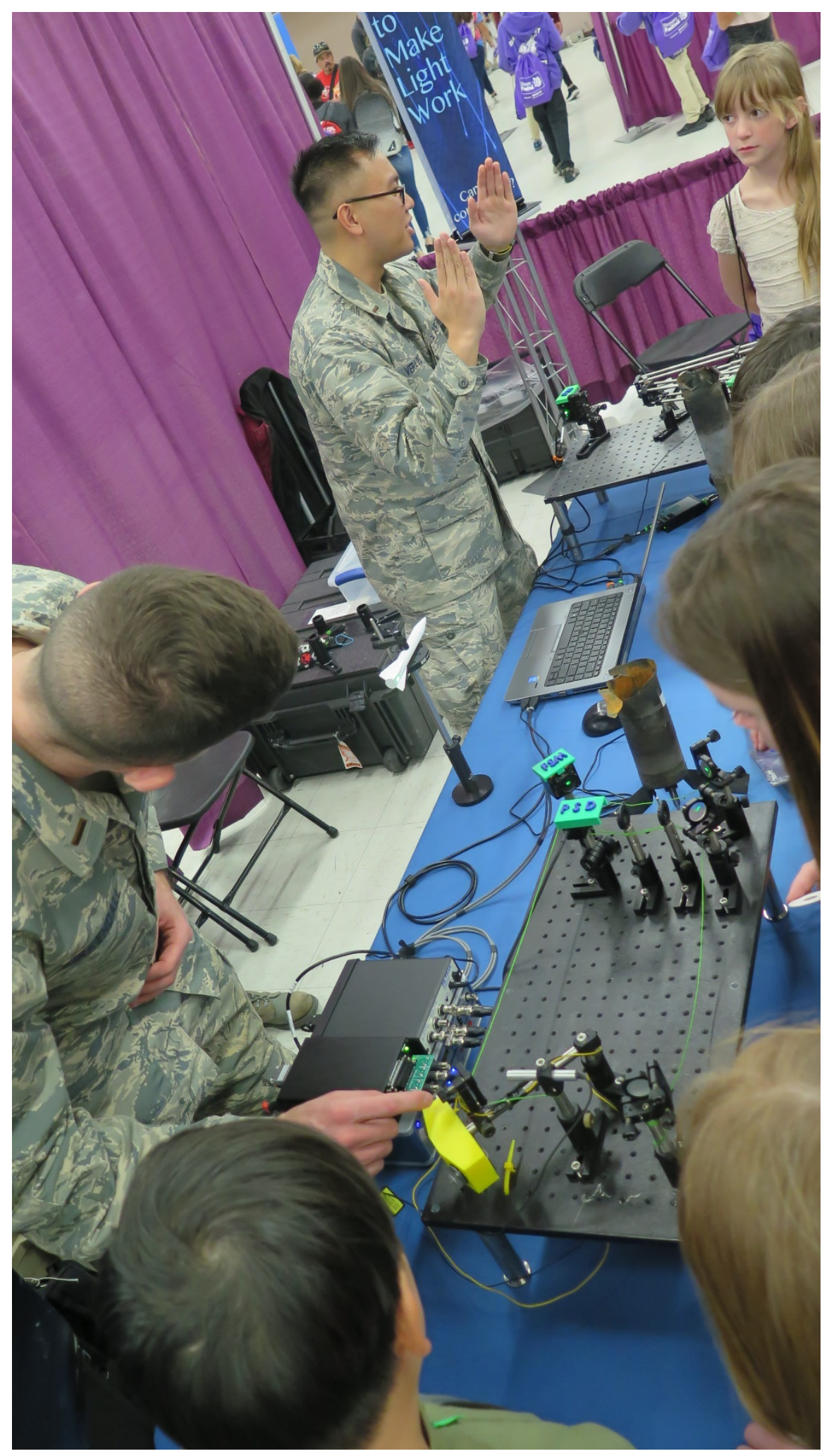

Figure 2. Lieutenants Kirkendall and Vera Cruz showcase the demo at an Albuquerque Big Brothers Big Sisters Event 


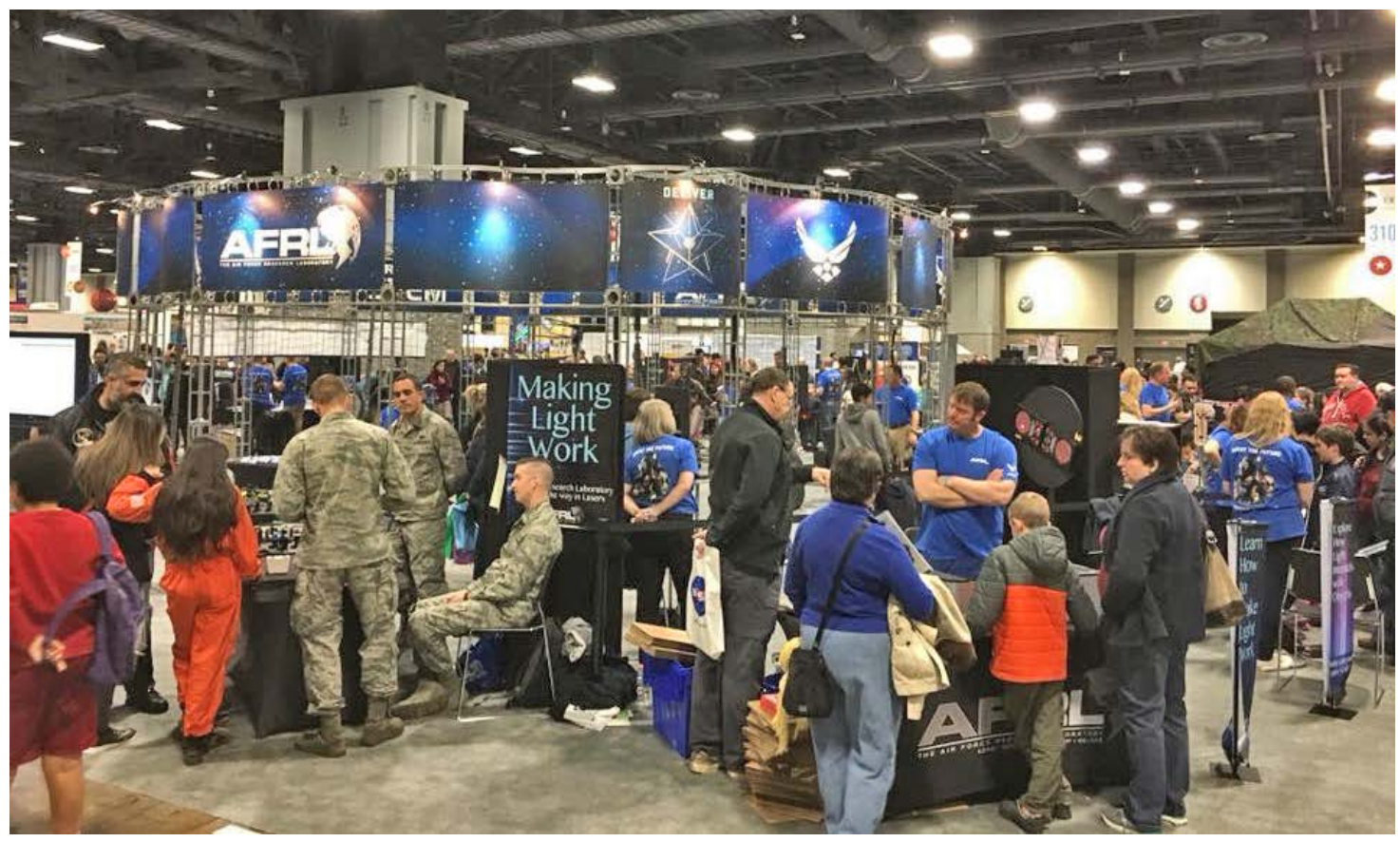

Figure 3. Lieutenants Threlkeld, Brewer, and Taylor took the demo to the 2018 US Science and Engineering Festival in Washington DC

\section{SYSTEM DESIGN AND LESSONS LEARNED}

The LOS Demo's most important components are the FSM and PSD. Our system uses an Optics In Motion FSM, model OIM5001, 1-inch mirror. This is an older model and no longer being sold but a comparable replacement model is listed in the appendix with the full Bill of Materials. The PSD used is an On-Trak Position Sensing Module, PSM 2-3I connected to an OT-301 Position Sensing Amplifier. The PSD uses a 3.0 x $3.0 \mathrm{~mm}$ quadrant InGaAs detector and has a frequency response of DC to $15 \mathrm{kHz}$ (rage dependent). The general configuration of our bench is shown in the figure below.

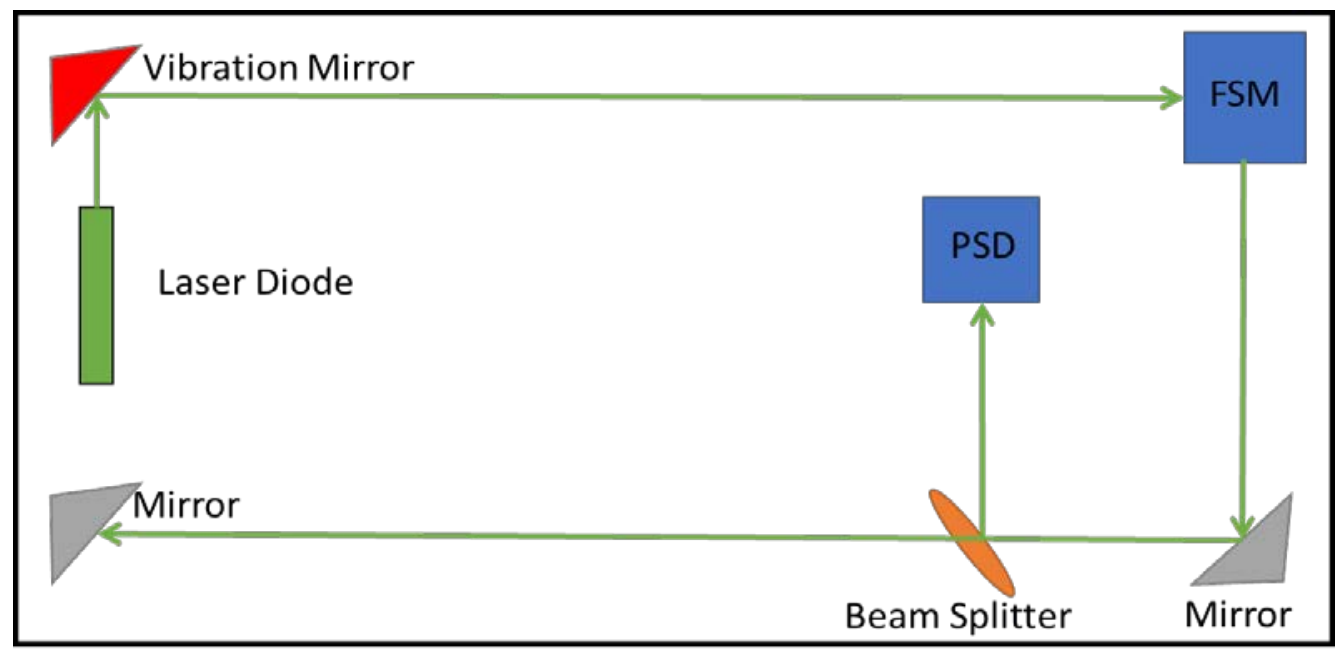

Figure 4. Diagram of the LOS Demo 
One of the first challenges we faced when designing this system was finding a controllable vibration source. Optics mounts and components are specifically designed to be vibration resistant and thus we had to be creative. Our first endeavor was to mount a small vibrating disc motor directly onto the side of a kinetic mirror mount using adhesive backing. While this was able to give us a small disturbance in one axis, it was barely enough jitter to see and was not the dramatic effect we were looking for. Even a pair of motors mounted perpendicular to each other was not enough to create the desired effect. We also had to replace the adhesive frequently which would have been a major logistical challenge to long term use. Our next attempt focused on the mirror mount. We figured a standard post mounted system wouldn't give us the large deflections needed and thus we would have to create a rigid, adjustable, yet vibration susceptible mount. Using a series of optical post mounting components, we created a cantilevered beam upon which to mount our mirror. A quick test of our disc motors showed they didn't have the power needed to move this larger system. We then decided to look around and find objects in everyday life that use intermittent vibration, hoping to discover a creative solution to our problem. Shortly thereafter we found exactly what we needed: the rumble motors in a video game controller. Modern controllers use a pair of unbalanced, offset motors to create a strong vibration effect. We scavenged a pair of these motors and checked the magnitude of the disturbance. It was perfect: strong enough to be easily visible but also small enough to be correctable by our system. We designed and 3D printed a custom holder to mount these directly onto our cantilevered post and wired them to our power supply. It worked perfectly!

\section{4: System Expansion and Modifications}

While this system provides a unique visual example of one of the key challenges faced by laser weapon systems, it obviously doesn't tell the whole story. As mentioned in the discussion of our most Frequently Asked Questions, the idea of target tracking was often conflagrated with this stabilization demo. Thus, the first major expansion our team undertook was the design and construction of a table top beam director. Our team designed and assembled a joystick controlled, open architecture beam director that allows audiences to see the differences between internal stabilization and distant target tracking. This beam director has been integrated with the LOS Stabilization Demo many times and had been greatly appreciated by many audiences. For information on the parts contained and schematics of this expansion please contact the authors of this paper.

The second expansion we intend to add is a visualization of the effect of beam spread. As previously described, jitter is a problem because it smears out laser power over a larger spot on the target than ideal. Our plan is to develop a photosensitive target board that records where the beam is hitting it and scores "points" based on how close you are to the center. These "points" represent accumulated energy and once enough energy has been accumulated, the target indicates destruction. Thus, users would be able to observe how fast one can achieve the desired effect given the presence of jitter when the stabilization loop is both off and on. A fun challenge after this would be to place the target board on a motorized rail and challenge the users to track it using our beam director while it moves on its "flight path".

While all of these demos are excellent, specific examples of the technology research we are doing, they may not seem particularly helpful for the classroom teacher who doesn't have several thousand dollars for a single demo. Thus, we developed several ways these effects can be shown using common household items. Simply having a student point a laser pointer at a small target spot clearly illustrates jitter. Add in a handheld mirror and you can show the issues with a control loop. By giving one student the mirror and the other the laser pointer you have induced jitter in both sides of the system. Instructing the "source" to hold the beam steady while the "mirror" is instructed to aim the beam at different points on the wall. From this simple demo you can show the connection between linear distance and angular displacement (Geometry), reflectance angle off of a mirror (physics), and much more! In several instances I have taken a cheap handheld mirror, laser pointer, and wheeled chair to STEM outreach events. Having a group of three students work together to push the chair holding the source student (platform motion), aim the beam at the mirror (source stabilization), and use the mirror to aim at a target (beam director tracking) is a fun, interactive way to show these same concepts. Students often come up with fantastic questions 
after seeing these demos. For advanced students, the discussion can quickly move into feedback loops and basic control theory with a focus on the importance of latency, sensor range and the possibilities of prediction.

Even without fancy optics and laboratory grade equipment anyone can share the lessons and technology

fundamentals behind optical stabilization. All it takes is a bit of creativity and framing the discussion in a way that your audience can connect and engage with!

Proc. of SPIE Vol. 11143 111431D-7

Downloaded From: https://www.spiedigitallibrary.org/conference-proceedings-of-spie on 26 Apr 2023 Terms of Use: https://www.spiedigitallibrary.org/terms-of-use 